\title{
Erratum: Nucleon Matrix Elements of the Antisymmetric Quark Tensor [Phys. Rev. Lett. 122, 122001 (2019)]
}

\author{
Martin Hoferichter®, Bastian Kubis@, Jacobo Ruiz de Elvira๑, and Peter Stoffer®
}

(Q) (Received 16 April 2020; published 14 May 2020)

\begin{abstract}
We correct normalization and slope of the nucleon tensor form factors $F_{2, T}^{s, N}, F_{3, T}^{s, N}$ in the original Letter due to incorrect input for the strangeness form factors.

DOI: 10.1103/PhysRevLett.124.199901
\end{abstract}

The estimates for the normalization and slope of the form factors $F_{2, T}^{s, N}, F_{3, T}^{s, N}$ in [1] rely on input for the strangeness magnetic moment as well as electric and magnetic radii, $\mu^{s}=0.006(4),\left\langle r_{E, s}^{2}\right\rangle^{N}=0.0012(9) \mathrm{fm}^{2},\left\langle r_{M, s}^{2}\right\rangle^{N}=$ $0.0014(27) \mathrm{fm}^{2}$, as taken from [2]. However, these quantities include a charge factor $-1 / 3$, and accordingly should be multiplied by -3 to correspond to the standard conventions for nucleon strangeness form factors. The corrected values are given in Table I, together with an update from the more recent calculation [3], which finds $\mu^{s}=-0.017(4)$, $\left\langle r_{E, s}^{2}\right\rangle^{N}=-0.0048(6) \mathrm{fm}^{2},\left\langle r_{M, s}^{2}\right\rangle^{N}=-0.015(9) \mathrm{fm}^{2}$.

TABLE I. Normalizations and slopes for the nucleon tensor form factors $F_{2, T}^{s, N}, F_{3, T}^{s, N}$. The first two rows refer to the corrected input from [2], the last two rows to the more recent calculation [3].

\begin{tabular}{lccc}
\hline \hline Form factor & Normalization & Slope $\left[\mathrm{GeV}^{-2}\right]$ & Strangeness input \\
\hline$F_{2, T}^{s, N}$ & $0.009(10)$ & $0.011(23)$ & {$[2]$} \\
$F_{3, T}^{s, N}$ & $-0.005(5)$ & $-0.002(12)$ & $0.041(26)$ \\
$F_{2, T}^{s, N}$ & $0.009(5)$ & $-0.015(13)$ & {$[3]$} \\
$F_{3, T}^{s, N}$ & $-0.004(3)$ & & \\
\hline \hline
\end{tabular}

We thank Constantia Alexandrou for correspondence on the conventions of $[2,3]$.

[1] M. Hoferichter, B. Kubis, J. Ruiz de Elvira, and P. Stoffer, Phys. Rev. Lett. 122, 122001 (2019).

[2] C. Alexandrou, M. Constantinou, K. Hadjiyiannakou, K. Jansen, C. Kallidonis, G. Koutsou, and A. Vaquero Avilés-Casco, Phys. Rev. D 97, 094504 (2018).

[3] C. Alexandrou, S. Bacchio, M. Constantinou, J. Finkenrath, K. Hadjiyiannakou, K. Jansen, and G. Koutsou, Phys. Rev. D 101, 031501(R) (2020).

Published by the American Physical Society under the terms of the Creative Commons Attribution 4.0 International license. Further distribution of this work must maintain attribution to the author(s) and the published articles title, journal citation, and DOI. 